\title{
Special Section Guest Editorial: Metamaterials: Fundamentals and Applications
}

\author{
Allan D. Boardman ${ }^{a}$ and Mikhail Noginov ${ }^{b}$ \\ ${ }^{a}$ University of Salford, Joule Physics Laboratory, Salford, UK \\ a.d.boardman@salford.ac.uk \\ ${ }^{\mathrm{b}}$ Norfolk State University number 700 Park Avenue, Norfolk, Virginia, USA \\ mnoginov@vger.nsu.edu
}

Metamaterials have attracted a lot of attention over the last decade ${ }^{1}$ and are destined to be the generic basis of applications in many areas of life. They are artificial composites and can be constructed in a variety of ways using some forms of artificial atoms and molecules, which can be termed metaparticles.

In the beginning, the emphasis was on microwaves, and a metaparticle consisted of a metal wire combined with a metal split ring. If such metaparticles are arranged at a distance apart greater than their size, then the emergent bulk metamaterial is an average, effective medium that can display a negative permittivity and acquire magnetic properties that have a negative permeability. It is recognised now that many combinations of these negative properties can be created and this beautiful combination of electric and magnetic properties has stimulated a vast global interest. ${ }^{2,3}$ Indeed, there is now a major effort to create materials suitable for the optical frequency range and there is, therefore, a major interest in all aspects of how to deploy nanoparticles, for metamaterial purposes, as opposed to wires and split rings. The latter could be scaled down with considerable care and effort but, in general, many new metamaterials will be collections of nanoparticle dispersions, leading to fascinating, collective, average behaviour. In this context, SPIE hosts the Optics and Photonics conference each year in San Diego, California. Considered the largest international meeting of its type in North America, it contains a significant number of constituent conferences on nanoscience and nanoengineering. It is a pleasure to present some pivotal papers from these conferences devoted to metamaterials and metallic nanostructures here as a special section to this journal.

\section{References}

1. A. D. Boardman, N. King, and L. Velasco, "Negative Refraction in Perspective," Electromagnetics 25, 365-389 (2005).

2. K. L. Tsakmakidis, A. D. Boardman, and O. Hess, “"Trapped rainbow' storage of light in metamaterials," Nature (London) 450, 397-401 (2007).

3. A. D. Boardman, V. V. Grimalsky, Y. S. Kivshar, S. V. Koshevaya, M. Lapine, N. M. Litchinitser, V. N. Malnev, M. Noginov, Y. G. Rapoport, and V. M. Shalaev, "Active and tunable metamaterials," Laser Photonics Rev. 5, 287-307 (2011).

$1934-2608 / 2011 / \$ 25.00$ @ 2011 SPIE 